\title{
HUREWICZ THEOREM FOR ASSOUAD-NAGATA DIMENSION
}

\author{
N. BRODSKIY, J. DYDAK, M. LEVIN, AND A. MITRA
}

\begin{abstract}
Given a function $f: X \rightarrow Y$ of metric spaces, its asymptotic dimension $\operatorname{asdim}(f)$ is the supremum of $\operatorname{asdim}(A)$ such that $A \subset X$ and $\operatorname{asdim}(f(A))=0$. Our main result is
\end{abstract}

Theorem 0.1. $\operatorname{asdim}(X) \leq \operatorname{asdim}(f)+\operatorname{asdim}(Y)$ for any large scale uniform function $f: X \rightarrow Y$.

0.1 generalizes a result of Bell and Dranishnikov [3] in which $f$ is Lipschitz and $X$ is geodesic. We provide analogs of 0.1 for AssouadNagata dimension $\operatorname{dim}_{A N}$ and asymptotic Assouad-Nagata dimension $\operatorname{asdim}_{A N}$. In case of linearly controlled asymptotic dimension l-asdim we provide counterexamples to three questions of Dranishnikov [14].

As an application of analogs of 0.1 we prove

Theorem 0.2. If $1 \rightarrow K \rightarrow G \rightarrow H \rightarrow 1$ is an exact sequence of groups and $G$ is finitely generated, then

$$
\operatorname{asdim}_{A N}\left(G, d_{G}\right) \leq \operatorname{asdim}_{A N}\left(K, d_{G} \mid K\right)+\operatorname{asdim}_{A N}\left(H, d_{H}\right)
$$

for any word metrics metrics $d_{G}$ on $G$ and $d_{H}$ on $H$.

0.2 extends a result of Bell and Dranishnikov [3] for asymptotic dimension.

\section{Contents}

1. Introduction 2

2. Ostrand theorem for asymptotic dimension 2

3. Components, dimension, and coarseness 5

4. Dimension of a function 8

Date: May 17, 2006.

1991 Mathematics Subject Classification. Primary: 54F45, 54C55, Secondary: 54E35, 18B30, 54D35, 54D40, 20H15.

Key words and phrases. Asymptotic dimension, coarse category, Lipschitz functions, Nagata dimension.

The second-named and third-named authors were partially supported by Grant No.2004047 from the United States-Israel Binational Science Foundation (BSF), Jerusalem, Israel. 
5. Asymptotic dimension of groups 12

6. Linearly controlled asymptotic dimension of groups 14

7. Assouad-Nagata dimension 16

8. Asymptotic Assouad-Nagata dimension 16

$\begin{array}{ll}\text { References } & 18\end{array}$

\section{INTRODUCTION}

The well-known Hurewicz Theorem for maps (also known as DimensionLowering Theorem, see [18], Theorem 1.12 .4 on p.109) says $\operatorname{dim}(X) \leq$ $\operatorname{dim}(f)+\operatorname{dim}(Y)$ if $f: X \rightarrow Y$ is a closed map of separable metric spaces and $\operatorname{dim}(f)$ is defined as the supremum of $\operatorname{dim}\left(f^{-1}(y)\right)$, $y \in Y$. Bell and Dranishnikov [3] proved a variant of Hurewicz Theorem for asymptotic dimension without defining the asymptotic dimension of a function. However, Theorem 1 of [3] may be restated as $\operatorname{asdim}(X) \leq \operatorname{asdim}(f)+\operatorname{asdim}(Y)$, where $\operatorname{asdim}(f)$ is the smallest integer $n$ such that asdim $\left(f^{-1}\left(B_{R}(y)\right)\right) \leq n$ uniformly for all $R>0$. As an application it is shown in [3] that $\operatorname{asdim}(G) \leq \operatorname{asdim}(K)+\operatorname{asdim}(H)$ for any exact sequence $1 \rightarrow K \rightarrow G \rightarrow H \rightarrow 1$ of finitely generated groups. That inequality was extended subsequently by Dranishnikov and Smith [16] to all countable groups.

The purpose of this paper is to generalize Hurewicz Theorem to variants of asymptotic dimension: asymptotic Assouad-Nagata dimension and Assouad-Nagata dimension. In the process we produce a much simpler proof than that in [3] and a stronger result: the function is only assumed to be large scale uniform instead of Lipschitz, and the domain is not required to be geodesic.

One of the main tools is Kolmogorov's idea used in his solution to Hilbert's 13th Problem. In dimension theory it is known as Ostrand Theorem. Another tool is reformulating Gromov's [19] definition of asymptotic dimension in terms of $r$-components of spaces. That leads to a definition of asymptotic dimension of a function in terms of doubleparameter components, a concept well-suited for Kolmogorov Trick.

\section{OSTRAND THEOREM FOR ASYMPTOTIC DIMENSION}

The aim of this section is to prove a variant of Ostrand's Theorem for large scale dimensions. As an application we present a simple proof of the Logarithmic Law for large scale dimensions.

Intuitively, a metric space is of dimension 0 at scale $r$ if it can be represented as a collection of $r$-disjoint and uniformly bounded subsets. The following definition of Gromov [19] defines asymptotic dimension 
at most $n$ in terms of being represented, for each $r>0$, as the union of $n+1$ sets of dimension 0 at scale $r$. That corresponds to the well-known property of topologically $n$-dimensional spaces.

Definition 2.1. A metric space $X$ is said to be of asymptotic dimension at most $n$ (notation: $\operatorname{asdim}(X) \leq n$ ) if there is a function $D_{X}: R_{+} \rightarrow$ $R_{+}$such that for all $r>0$ there is a cover $\mathcal{U}=\bigcup_{i=1}^{n+1} \mathcal{U}_{i}$ of $X$ so that each $\mathcal{U}_{i}$ is $r$-disjoint (that means $d(a, b) \geq r$ for any two points $a$ and $b$ belonging to different elements of $\left.\mathcal{U}_{i}\right)$ and the diameter of elements of $\mathcal{U}$ is bounded by $D_{X}(r)$.

We refer to the function $D_{X}$ as an $n$-dimensional control function for $X$. When discussing variants of asymptotic dimension it is convenient to allow $D_{X}$ to assume infinity as its value at some range of $r$.

Definition 2.2. A metric space $X$ is said to be of Assouad-Nagata dimension (see [20] and [7]) at most $n$ (notation: $\operatorname{dim}_{A N}(X) \leq n$ ) if it has an $n$-dimensional control function $D_{X}$ that is a dilation $\left(D_{X}(r)=\right.$ $c \cdot r$ for some $c>0)$.

A metric space $X$ is said to be of asymptotic Assouad-Nagata dimension at most $n$ (notation: $\operatorname{asdim}_{A N}(X) \leq n$ ) if it has an $n$-dimensional control function $D_{X}$ that is linear $\left(D_{X}(r)=c \cdot r+b\right.$ for some $\left.b, c \geq 0\right)$.

A metric space $X$ is said to be of linearly controlled asymptotic dimension at most $n$ (Dranishnikov [14], notation: 1 -asdim $(X) \leq n$ ) if it has an $n$-dimensional control function $D_{X}$ satisfying $D_{X}(r)=c \cdot r$ for some $c>0$ and for all $r$ belonging to some unbounded subset of $R_{+}$. Strictly speaking, the original definition of Dranishnikov [14] is formulated in terms of Lebesque numbers. However, just as in the case of asymptotic dimension, it is equivalent to our definition.

A metric space $X$ is said to be of microscopic Assouad-Nagata dimension at most $n$ if it has an $n$-dimensional control function $D_{X}: R_{+} \rightarrow$ $R_{+} \cup \infty$ that is a dilation near $0: D_{X}(r)=c \cdot r$ for some $c>0$ and all $r$ smaller than some positive number $M, D_{X}(r)=\infty$ for all $r \geq M$.

Definition 2.3. Given a metric space $X$ and $k \geq n+1 \geq 1$ an $(n, k)$ dimensional control function for $X$ is a function $D_{X}: R_{+} \rightarrow R_{+}$such that for any $r>0$ there is a family $\mathcal{U}=\bigcup_{i=1}^{k} \mathcal{U}_{i}$ satisfying the following conditions:

(1) each $\mathcal{U}_{i}$ is $r$-disjoint,

(2) each $\mathcal{U}_{i}$ is $D_{X}(r)$-bounded, 
(3) each element $x \in X$ belongs to at least $k-n$ elements of $\mathcal{U}$ (equivalently, $\bigcup_{i \in T} \mathcal{U}_{i}$ is a cover of $X$ for every subset $T$ of $\{1, \ldots, k\}$ consisting of $n+1$ elements).

The following result is an adaptation of a theorem by Ostrand [25].

Theorem 2.4. If $D_{X}^{(n+1)}$ is an $n$-dimensional control function of $X$ and one defines a sequence of functions $\left\{D_{X}^{(i)}\right\}_{i \geq n+1}$ inductively by $D_{X}^{(i+1)}(r)=$ $D_{X}^{(i)}(3 r)+2 r$ for all $i \geq n+1$, then each $D_{X}^{(k)}$ is an $(n, k)$-dimensional control function of $X$ for all $k \geq n+1$.

Proof. The proof is by induction on $k$. The case of $k=n+1$ is obvious. Suppose the result holds for some $k \geq n+1$. Let $\mathcal{U}=\bigcup_{i=1}^{k} \mathcal{U}_{i}$ be a family such that each $\mathcal{U}_{i}$ is $3 r$-disjoint, each $\mathcal{U}_{i}$ is $D_{X}^{(k)}(3 r)$-bounded, and each element $x \in X$ belongs to at least $k-n$ elements of $\mathcal{U}$. Define $\mathcal{U}_{i}^{\prime}$ to be the $r$-neighborhoods of elements of $\mathcal{U}_{i}$ for $i \leq k$. Notice elements of $\mathcal{U}_{i}^{\prime}$ are $\left(D_{X}^{(k)}(3 r)+2 r\right)$-bounded and are $r$-disjoint. Define $\mathcal{U}_{k+1}^{\prime}$ as the collection of all sets of the form $\bigcap_{s \in S} A_{s} \backslash \bigcup_{i \notin S} \mathcal{U}_{i}^{\prime}$, where $S$ is a subset of $\{1, \ldots, k\}$ consisting of exactly $k-n$ elements and $A_{s} \in \mathcal{U}_{s}$.

Notice that any element of $\mathcal{U}_{k+1}^{\prime}$ is contained in a single element of some $\mathcal{U}_{j}$. Thus elements of each $\mathcal{U}_{i}^{\prime}$ are $\left(D_{f}^{(k)}(3 r)+2 r\right)$-bounded.

Given two different sets $A=\bigcap_{s \in S} A_{s} \backslash \bigcup_{i \notin S} \mathcal{U}_{i}^{\prime}$, where $S$ is a subset of $\{1, \ldots, k\}$ consisting of exactly $k-n$ elements and $A_{s} \in \mathcal{U}_{s}$, and $B=\bigcap_{t \in T} B_{t} \backslash \bigcup_{i \notin T} \mathcal{U}_{i}^{\prime}$, where $T$ is a subset of $\{1, \ldots, k\}$ consisting of exactly $k-n$ elements and $B_{t} \in \mathcal{U}_{t}$, we need to show $A$ and $B$ are $r$-disjoint. It is clearly so if $S=T$, so assume $S \neq T$. If $a \in A, b \in B$, and $d(a, b)<r$, then there is $s \in S \backslash T$ such that $a \in A_{s}$ prompting $b \in U \in \mathcal{U}_{s}^{\prime}$, a contradiction.

Suppose $x \in X$ belongs exactly to $k-n$ sets $\bigcup \mathcal{U}_{i}^{\prime}, i \leq k$, and let $S=\left\{i \leq k \mid x \in \bigcup \mathcal{U}_{i}^{\prime}\right\}$. If $x \notin \bigcup \mathcal{U}_{k+1}^{\prime}$, then $x$ must belong to $\bigcup \mathcal{U}_{j}^{\prime}$ for some $j \notin S$, a contradiction. Thus each $x \in X$ belongs to at least $k+1-n$ elements of $\left\{\bigcup \mathcal{U}_{i}^{\prime}\right\}_{i=1}^{k+1}$.

The product theorem for asymptotic dimension was proved in [15] using maps to polyhedra. The product theorem for Nagata dimension was proved in [20] using Lipschitz maps to polyhedra. Below we use Theorem 2.4 to give a simplified proof for all dimension theories. Note the metric on $X \times Y$ is the sum of corresponding metrics on $X$ and $Y$. 
Theorem 2.5. If $X$ and $Y$ are metric spaces, then

$$
D(X \times Y) \leq D(X)+D(Y)
$$

where $D$ stands for any of the following dimension theories: asymptotic dimension, asymptotic Assouad-Nagata dimension, Assouad-Nagata dimension, or microscopic Assouad-Nagata dimension.

Proof. Let $D(X)=m, D(Y)=n$ and let $k=m+n+1$. Pick $(m, k)$ dimension control function $D_{X}$ of $X$ and $(n, k)$-dimensional control function $D_{Y}$ of $Y$, both of the correct type (arbitrary, linear, dilation, or a dilation near 0 ). There are families $\left\{\mathcal{U}_{i}\right\}_{i=1}^{k}$ in $X$ and $\left\{\mathcal{V}_{i}\right\}_{i=1}^{k}$ in $Y$ that are $r$-disjoint and bounded by $D_{X}(r)$ and $D_{Y}(r)$ respectively, that cover $X$ and $Y$ at least $k-m$ times and $k-n$ times respectively. Then the family $\left\{\mathcal{U}_{i} \times \mathcal{V}_{i}\right\}_{i=1}^{k}$ covers $X \times Y$, as for any point $(x, y), x$ is contained in sets from at least $k-m=n+1$ families from $\left\{\mathcal{U}_{i}\right\}_{i=1}^{k}$ and $y$ is contained in sets from at least $k-n=m+1$ families from $\left\{\mathcal{V}_{i}\right\}_{i=1}^{k}$, so there is at least one index $j$ such that $x$ is covered by $\mathcal{U}_{j}$ and $y$ is covered by $\mathcal{V}_{j}$. The family $\left\{\mathcal{U}_{i} \times \mathcal{V}_{i}\right\}_{i=1}^{k}$ is $r$-disjoint and is bounded by $D_{X}(r)+D_{Y}(r)$.

\section{Components, Dimension, And COARseness}

In this section we replace the language of $r$-disjoint families by the language of $r$-components. This language has the advantage of being portable to functions. It also allows for simple proofs of known results 3.11-3.12.

Definition 3.1. Let $f: X \rightarrow Y$ be a function of metric spaces, $A$ is a subset of $X$, and $r_{X}, r_{Y}$ are two positive numbers.

$A$ is $\left(r_{X}, r_{Y}\right)$-bounded if for any points $x, x^{\prime} \in A$ we have

$$
d_{X}\left(x, x^{\prime}\right) \leq r_{X} \quad \text { and } \quad d_{Y}\left(f(x), f\left(x^{\prime}\right)\right) \leq r_{Y} .
$$

An $\left(r_{X}, r_{Y}\right)$-chain in $A$ is a sequence of points $x_{1}, \ldots, x_{k}$ in $A$ such that for every $i<k$ the set $\left\{x_{i}, x_{i+1}\right\}$ is $\left(r_{X}, r_{Y}\right)$-bounded.

$A$ is $\left(r_{X}, r_{Y}\right)$-connected if for any points $x, x^{\prime} \in A$ can be connected in $A$ by an $\left(r_{X}, r_{Y}\right)$-chain.

Notice that any subset $A$ of $X$ is a union of its $\left(r_{X}, r_{Y}\right)$-components (the maximal $\left(r_{X}, r_{Y}\right)$-connected subsets of $A$ ).

Definition 3.2. Let $f: X \rightarrow Y$ be a function of metric spaces. $f$ is called large scale uniform if there is function $c_{f}: R_{+} \rightarrow R_{+}$such that $d_{X}(x, y) \leq r$ implies $d_{Y}(f(x), f(y)) \leq c_{f}(r)$. The function $c_{f}$ will be called a coarseness control function of $f$. 
Notice $f$ is Lipschitz if and only if it has a coarseness control function that is a dilation. $f$ is asymptotically Lipschitz if and only if it has a coarseness control function that is linear.

The following Lemma describes a useful case in which double parameter components coincide with single parameter components. The proof of this Lemma is an easy exercise.

Lemma 3.3. Let $f: X \rightarrow Y$ be a function of metric spaces. $c_{f}: R_{+} \rightarrow$ $R_{+}$is a coarseness control function of $f$ if and only if for any subset $A$ of $X$ its $\left(r, c_{f}(r)\right)$-components coincide with its $r$-components.

The following Lemma describes the way we construct a subset of $X$ with all $\left(r_{X}, r_{Y}\right)$-components being $\left(R_{X}, R_{Y}\right)$-bounded. The proof of this Lemma is an easy exercise.

Lemma 3.4. Let $f: X \rightarrow Y$ be a function of metric spaces, $B$ be a subset of $X$, and $A$ be a subset of $Y$. If all $r_{X}$-components of $B$ are $R_{X}$-bounded and all $r_{Y}$-components of $A$ are $R_{Y}$-bounded then all $\left(r_{X}, r_{Y}\right)$-components of the set $B \cap f^{-1}(A)$ are $\left(R_{X}, R_{Y}\right)$-bounded.

Lemma 3.5. Let $f: X \rightarrow Y$ be a function of metric spaces and $A, B$ be subsets of $X$. Suppose that all $\left(r_{X}^{A}, r_{Y}^{A}\right)$-components of $A$ are $\left(R_{X}^{A}, R_{Y}^{A}\right)$ bounded and all $\left(r_{X}^{B}, r_{Y}^{B}\right)$-components of $B$ are $\left(R_{X}^{B}, R_{Y}^{B}\right)$-bounded. If $R_{X}^{B}+2 r_{X}^{B}<r_{X}^{A}$ and $R_{Y}^{B}+2 r_{Y}^{B}<r_{Y}^{A}$ then all $\left(r_{X}^{B}, r_{Y}^{B}\right)$-components of $A \cup B$ are $\left(R_{X}^{A}+2 r_{X}^{A}, R_{Y}^{A}+2 r_{Y}^{A}\right)$-bounded.

Proof. Let $x=x_{1}, x_{2}, \ldots, x_{n}=x^{\prime}$ form an $\left(r_{X}^{B}, r_{Y}^{B}\right)$-chain in $A \cup B$.

Notice that, if for some indices $i<j$ we have $x_{k} \in B$ for all $i<k<j$, then $x_{i+1}$ and $x_{j-1}$ are in one $\left(r_{X}^{B}, r_{Y}^{B}\right)$-component of $B$ and therefore $d_{X}\left(x_{i+1}, x_{j-1}\right) \leq R_{X}^{B}$ and $d_{Y}\left(f\left(x_{i+1}\right), f\left(x_{j-1}\right)\right) \leq R_{Y}^{B}$.

If $x_{i}, x_{j} \in A$ and $x_{k} \in B$ for all $i<k<j$, then

$d_{X}\left(x_{i}, x_{j}\right) \leq d_{X}\left(x_{i}, x_{i+1}\right)+d_{X}\left(x_{i+1}, x_{j-1}\right)+d_{X}\left(x_{j-1}, x_{j}\right) \leq r_{X}^{B}+R_{X}^{B}+r_{X}^{B}<r_{X}^{A}$ and, similarly, $d_{Y}\left(f\left(x_{i}\right), f\left(x_{j}\right)\right) \leq r_{Y}^{B}+R_{Y}^{B}+r_{Y}^{B}<r_{Y}^{A}$. Thus the points $x_{i}, x_{j}$ belong to the same $\left(r_{X}^{A}, r_{Y}^{A}\right)$-component of $A$. This implies that all points in the chain $x=x_{1}, x_{2}, \ldots, x_{n}=x^{\prime}$ belonging to $A$ are in one $\left(r_{X}^{A}, r_{Y}^{A}\right)$-component of $A$.

Now let $x_{s}$ be the first point in the chain belonging to $A$ and $x_{t}$ be the last point in the chain belonging to $A$. Then

$$
\begin{gathered}
d_{X}\left(x, x^{\prime}\right) \leq \\
d_{X}\left(x_{1}, x_{s-1}\right)+d_{X}\left(x_{s-1}, x_{s}\right)+d_{X}\left(x_{s}, x_{t}\right)+d_{X}\left(x_{t}, x_{t+1}\right)+d_{X}\left(x_{t+1}, x_{n}\right) \leq \\
\leq R_{X}^{B}+r_{X}^{B}+R_{X}^{A}+r_{X}^{B}+R_{X}^{B}<R_{X}^{A}+2 r_{X}^{A} . \\
\text { Similarly, } d_{Y}\left(f(x), f\left(x^{\prime}\right)\right) \leq R_{Y}^{B}+r_{Y}^{B}+R_{Y}^{A}+r_{Y}^{B}+R_{Y}^{B}<R_{Y}^{A}+2 r_{Y}^{A} . \quad \square
\end{gathered}
$$


Corollary 3.6. Let $f: X \rightarrow Y$ be a function of metric spaces and $\left\{B_{i}\right\}_{i=1}^{n}$ be subsets of $X$. Suppose that for every $i$ all $\left(r_{X}^{(i)}, r_{Y}^{(i)}\right)$-components of $B_{i}$ are $\left(R_{X}^{(i)}, R_{Y}^{(i)}\right)$-bounded. If, for every $i<n, R_{X}^{(i+1)}+2 r_{X}^{(i+1)}<r_{X}^{(i)}$ and $R_{Y}^{(i+1)}+2 r_{Y}^{(i+1)}<r_{Y}^{(i)}$, then all $\left(r_{X}^{(n)}, r_{Y}^{(n)}\right)$-components of $\bigcup_{i=1}^{n} B_{i}$ are $\left(R_{X}^{(1)}+2 r_{X}^{(1)}, R_{Y}^{(1)}+2 r_{Y}^{(1)}\right)$-bounded.

Proof. By induction using Lemma 3.5.

Proposition 3.7. Suppose $A$ is a subset of a metric space $X, m \geq 0$, and $R>0$. If $D_{A}$ is an $m$-dimensional control function of $A$, then $D_{B}(x):=D_{A}(x+2 R)+2 R$ is an m-dimensional control function of the $R$-neighborhood $B=B(A, R)$ of $A$.

Proof. Given $r>0$ express $A$ as $\bigcup_{i=1}^{m+1} A_{i}$ such that $(r+2 R)$ components of $A_{i}$ are $D_{A}(r+2 R)$-bounded. Given an $r$-component of $B_{i}:=B\left(A_{i}, R\right)$, each point in that component is $R$-close to a single $(r+2 R)$-component of $A_{i}$. Therefore $r$-components of $B_{i}$ are $\left(D_{A}(r+2 R)+2 R\right)$-bounded.

Definition 3.8. Given a metric space $X$ and $r>0$ the $r$-scale $d i$ mension $\mathrm{r}-\operatorname{dim}(X)$ is the smallest integer $n \geq 0$ such that $X$ can be expressed as $X=\bigcup_{i=1}^{n+1} X_{i}$ and $r$-components of each $X_{i}$ are uniformly bounded.

Notice $\operatorname{asdim}(X)$ is the smallest integer $n$ such that $\mathrm{r}-\operatorname{dim}(X) \leq n$ for all $r>0$. Also, $\operatorname{asdim}(X)$ is the smallest integer such that for each $r>0$ the space $X$ can be expressed as $X=\bigcup_{i=1}^{n+1} X_{i}$ so that r-dim $\left(X_{i}\right) \leq 0$ for each $i \leq n+1$.

Corollary 3.9. Suppose $X$ is a metric space. If, for every $r>0$, there is a subspace $X_{r}$ of $X$ such that $\operatorname{asdim}\left(X_{r}\right) \leq n$ and $r$-dim $\left(X \backslash X_{r}\right) \leq n$, then $\operatorname{asdim}(X) \leq n$.

Proof. Express $X \backslash X_{r}$ as $\bigcup_{\substack{i=1 \\ n+1}}^{n+1} A_{i}$ such that $r$-components of $A_{i}$ are $R$-bounded. Express $X_{r}$ as $\bigcup_{i=1}^{n+1} B_{i}$ so that $(R+2 r)$-components of $B_{i}$ are $M$-bounded. By Lemma 3.5, r-components of $A_{i} \cup B_{i}$ are $(M+2 R+4 r)$-bounded. 
Corollary 3.10. Suppose $G$ is a group with a left-invariant metric and $G_{r}$ is the subgroup of $G$ generated by $B\left(1_{G}, r\right)$. If, for every $r>0$, $\operatorname{asdim}\left(G_{r}\right) \leq n$, then $\operatorname{asdim}(G) \leq n$.

Proof. Consider $g_{s} \in G, s \in S$, so that $\left[g_{s}\right]$ enumerate $G / G_{r} \backslash\left[G_{r}\right]$. Notice $Y=\bigcup_{s \in S} g_{s} \cdot G_{r}$ is of $\mathrm{r}-\operatorname{dim}(Y) \leq \operatorname{asdim}\left(G_{r}\right)$ and $Y \cup G_{r}=G$.

Corollary 3.11 (Dranishnikov-Smith [16]). If $G$ is a group with a proper left-invariant metric, then asdim $(G)$ is the supremum of asdim $(H)$ over all finitely generated subgroups $H$ of $G$.

Proof. Since balls $B\left(1_{G}, r\right)$ are finite, the groups $G_{r}$ in 3.10 are finitely generated.

Corollary 3.12 (Bell-Dranishnikov [3]). Suppose $X$ is a metric space and $\left\{X_{s}\right\}_{s \in S}$ is a family of subsets of $X$ such that $X=\bigcup_{s \in S} X_{s}$ and there is a single $n$-dimensional control function $D$ for all $X_{s}$. If, for every $r>0$, there is a subspace $X_{r}$ of $X$ such that asdim $\left(X_{r}\right) \leq n$ and the family $\left\{X_{s} \backslash X_{r}\right\}_{s \in S}$ is r-disjoint, then $\operatorname{asdim}(X) \leq n$.

Proof. Notice r-dim $\left(X \backslash X_{r}\right) \leq n$.

\section{Dimension of A FUnCtion}

When trying to generalize Hurewicz Theorem from covering dimension to any other dimension theory, the issue arises of how to define the dimension of a function. Let us present an example of a Lipschitz function demonstrating that replacing $\sup \left\{\operatorname{dim}\left(f^{-1}(y)\right) \mid y \in Y\right\}$ by $\sup \left\{\operatorname{asdim}\left(f^{-1}(B)\right) \mid B \subset Y\right.$ is bounded $\}$ does not work.

Proposition 4.1. There is a Lipschitz function $f: X \rightarrow Y$ of metric spaces such that

$$
\operatorname{asdim}(Y)=0=\sup \left\{\operatorname{asdim}\left(f^{-1}(B)\right) \mid B \subset Y \text { is bounded }\right\}
$$

and $\operatorname{asdim}(X)>0$.

Proof. Let $Y$ consist of points $2^{n}, n \geq 1$, on the $x$-axis and $X$ is the union of vertical segments $I_{n}$ of length $n$ and starting at $2^{n} . f: X \rightarrow Y$ is the projection. Since $f^{-1}(B)$ is bounded for every bounded $B \subset Y$, $\sup \left\{\operatorname{asdim}\left(f^{-1}(B)\right) \mid B \subset Y\right.$ is bounded $\}=0 . \quad$ Also, $\operatorname{asdim}(Y)=0$. However, $\operatorname{asdim}(X)>0$ as for each $n$ it has $n$-components of arbitrarily large size.

Remark 4.2. 4.1 shows the answer to a problem of Dranishnikov [14] in negative. That problem asks if $l-\operatorname{asdim}(X) \leq l-\operatorname{asdim}(Y)+1-\operatorname{asdim} f^{-1}$, where 1 -asdim $f^{-1}$ is defined as $\sup \left\{1-\operatorname{asdim}\left(f^{-1}(B)\right) \mid B \subset Y\right.$ is bounded $\}$ 
and $f$ is Lipschitz (see 2.2 for the definition of l-asdim). Notice l-asdim $(Y)=$ $0,1-\operatorname{asdim} f^{-1}=0$, and $1-\operatorname{asdim}(X) \geq \operatorname{asdim}(X)>0$ in 4.1 .

In view of 4.1 we define asymptotic dimension of a function as follows:

Definition 4.3. Given a function $f: X \rightarrow Y$ of metric spaces we define the asymptotic dimension $\operatorname{asdim}(f)$ of $f$ as the supremum of asymptotic dimensions of $A \subset X$ so that $f(A) \subset Y$ is of asymptotic dimension 0 .

Definition 4.4. Given a function $f: X \rightarrow Y$ of metric spaces and given $m \geq 0$, an $m$-dimensional control function of $f$ is a function $D_{f}: R_{+} \times R_{+} \rightarrow R_{+}$such that for all $r_{X}>0$ and $R_{Y}>0$ any $\left(\infty, R_{Y}\right)$ bounded subset $A$ of $X$ can be expressed as the union of $m+1$ sets whose $r_{X}$-components are $D_{f}\left(r_{X}, R_{Y}\right)$-bounded.

Proposition 4.5. Suppose $f: X \rightarrow Y$ is a function of metric spaces and $m \geq 0$. If asdim $(f) \leq m$, then $f$ has an $m$-dimensional control function $D_{f}$.

Proof. Fix $r_{X}>0$ and $R_{Y}>0$. Suppose for each $n$ there is $y_{n} \in Y$ such that $A_{n}=f^{-1}\left(B\left(y_{n}, R_{Y}\right)\right)$ cannot be expressed as the union of $m+1$ sets whose $r_{X}$-components are $n$-bounded. The set $C=\bigcup_{n=1}^{\infty} B\left(y_{n}, R_{Y}\right)$ cannot be bounded as $\operatorname{asdim}\left(f^{-1}(C)\right) \leq m$ for any bounded subset $C$ of $Y$. By passing to a subsequence we may arrange $y_{n} \rightarrow \infty$ and $\operatorname{asdim}(C)=0$, a contradiction.

Definition 4.6. Given a function $f: X \rightarrow Y$ of metric spaces and given $k \geq m+1 \geq 1$, an $(m, k)$-dimensional control function of $f$ is a function $D_{f}: R_{+} \times R_{+} \rightarrow R_{+}$such that for all $r_{X}>0$ and $R_{Y}>0$ any $\left(\infty, R_{Y}\right)$-bounded subset $A$ of $X$ can be expressed as the union of $k$ sets $\left\{A_{i}\right\}_{i=1}^{k}$ whose $r_{X}$-components are $D_{f}\left(r_{X}, R_{Y}\right)$-bounded so that any $x \in A$ belongs to at least $k-m$ elements of $\left\{A_{i}\right\}_{i=1}^{k}$.

Proposition 4.7. Let $f: X \rightarrow Y$ be a function of metric spaces and $m \geq 0$. Suppose there is an $m$-dimensional control function $D_{f}^{(m+1)}: R_{+} \times$ $R_{+} \rightarrow R_{+}$of $f$. If one defines inductively functions $D_{f}^{(k)}$ for $k>m+1$ by $D_{f}^{(k)}\left(r_{X}, R_{Y}\right)=D_{f}^{(k-1)}\left(3 r_{X}, R_{Y}\right)+2 r_{X}$, then each $D_{f}^{(k)}$ is an $(m, k)$ dimensional control function of $f$.

Proof. The proof is by induction on $k$. The case of $k=m+1$ is obvious. Suppose the result holds for some $k \geq n+1$ and a subset $A$ of $X$ is $\left(\infty, R_{Y}\right)$-bounded. There are $k$ subsets $\left\{A_{i}\right\}_{i=1}^{k}$, each has $3 r_{X^{-}}$-components bounded by $D_{f}^{(k)}\left(3 r_{X}, R_{Y}\right)$ such that the union of any $m+1$ of those sets covers $A$. 
Define $A_{i}^{\prime}$ to be the $r_{X^{-}}$-neighborhood of $A_{i}$ in $A$ for $i \leq k$. Notice $r_{X}$-components of each $A_{i}^{\prime}$ are $\left(D_{f}^{(k)}\left(3 r_{X}, R_{Y}\right)+2 r_{X}\right)$-bounded as they are contained in $r_{X}$-neighborhoods of $3 r_{X}$-components of $A_{i}$.

Define $A_{k+1}^{\prime}$ as the union of all sets $\bigcap_{i \in S} A_{i} \backslash \bigcup_{i \notin S} A_{i}^{\prime}$, where $S$ is a subset of $\{1, \ldots, k\}$ consisting of exactly $k-m$ elements.

Suppose $x \in A$ belongs exactly to $k-m$ sets $A_{i}$ such that $i \leq k$ and let $S=\left\{i \leq k \mid x \in A_{i}\right\}$. If $x \notin A_{k+1}^{\prime}$, then $x$ must belong to $A_{j}^{\prime}$ for some $j \notin S$. Thus each $x \in A$ belongs to at least $k+1-m$ elements of $\left\{A_{i}^{\prime}\right\}_{i=1}^{k+1}$.

Notice that any $r_{X^{-}}$-component of $A_{k+1}^{\prime}$ is contained in a single $r_{X^{-}}$ component of some $A_{j}$ resulting in $r_{X^{-}}$-components of each $A_{i}^{\prime}$ being $\left(D_{f}^{(k)}\left(3 r_{X}, R_{Y}\right)+2 r_{X}\right)$-bounded.

Proposition 4.8. Let $f: X \rightarrow Y$ be a function of metric spaces and $k, m \geq 0$. If $D_{f}: R_{+} \times R_{+} \rightarrow R_{+}$is an $(m, k)$-dimensional control function of $f$, then for any $B \subset Y$ whose $r_{Y}$-components are $R_{Y}$-bounded, the set $f^{-1}(B)$ can be covered by $k$ sets whose $\left(r_{X}, r_{Y}\right)$-components are $D_{f}\left(r_{X}, R_{Y}\right)$-bounded and every element of $f^{-1}(B)$ belongs to at least $k-m$ elements of that covering.

Proof. Given an $r_{Y}$-component $S$ of $B$ express $f^{-1}(S)$ as $A_{1}^{S} \cup \ldots \cup$ $A_{k}^{S}$ such that $r_{X}$-components of $A_{i}^{S}$ are $D_{f}\left(r_{X}, R_{Y}\right)$-bounded and every element of $f^{-1}(S)$ belongs to at least $k-m$ elements of that covering. Put $A_{i}=\bigcup_{S} A_{i}^{S}$ and notice each $\left(r_{X}, r_{Y}\right)$-component of $A_{i}$ is contained in an $r_{X}$-component of some $A_{i}^{S}$.

Theorem 4.9. Let $k=m+n+1$, where $m, n \geq 0$. Suppose $f: X \rightarrow Y$ is a large scale uniform function of metric spaces and asdim $(Y) \leq n$.

$$
\operatorname{asdim}(X) \leq m+n
$$

if there is an $(m, k)$-dimensional control function $D_{f}$ of $f$. Moreover, if one can choose the coarseness control function $c_{f}$ of $f$, the $n$-dimensional control function of $Y$, and $D_{f}$ to be linear (respectively, a dilation), then $X$ has a $(k-1)$-dimensional control function that is linear (respectively, a dilation).

Proof. Let $c_{f}$ be a coarseness control function of $f$. Let $D_{Y}: R_{+} \rightarrow R_{+}$ be an $(n, k)$-dimensional control function of $Y$. Notice we may require $c_{f}(r)>r, D_{Y}(r)>r$, and $D_{f}(r, R)>r+R$ as we may redefine those functions by adding $r$ or $r+R$ without losing their properties or type (linear, dilation or dilation near 0 ). 
Given a number $r>0$ we are going to construct a number $D_{X}(r)$ and represent the space $X$ as a union of $k$ sets $\left\{D^{j}\right\}_{j=1}^{k}$ with all $r$ components of $D^{j}$ being $D_{X}(r)$-bounded for every $j$.

Define inductively a sequence of numbers $r_{Y}^{(n+1)}<R_{Y}^{(n+1)}<r_{Y}^{(n)}<$ $R_{Y}^{(n)}<\cdots<r_{Y}^{(1)}<R_{Y}^{(1)}<r_{Y}^{(0)}<R_{Y}^{(0)}$ starting from $r_{Y}^{(n+1)}=c_{f}(r)$ and moving to lower indices so that for every $i$ we have $R_{Y}^{(i)}=D_{Y}\left(r_{Y}^{(i)}\right)$ and $r_{Y}^{(i)}=3 R_{Y}^{(i+1)}$.

Express $Y$ as the union of $k$ sets $\left\{A_{i}\right\}_{i=1}^{k}$ such that all $r_{Y}^{(0)}$-components of $A_{i}$ are $R_{Y}^{(0)}$-bounded for every $i$. Since $A_{i} \subset Y$, we can express the set $A_{i}$ as the union of $k$ sets $\left\{U_{i}^{j}\right\}_{j=1}^{k}$ such that all $r_{Y}^{(i)}$-components of $U_{i}^{j}$ are $R_{Y}^{(i)}$-bounded for every $j$ and every point $y \in A_{i}$ belongs to at least $m$ sets.

Define inductively a sequence of numbers $r_{X}^{(n+1)}<R_{X}^{(n+1)}<r_{X}^{(n)}<$ $R_{X}^{(n)} \cdots<r_{X}^{(1)}<R_{X}^{(1)}$ starting with $r_{X}^{(n+1)}=r$ and for every $i$ we have $R_{X}^{(i)}=D_{f}\left(r_{X}^{(i)}, R_{Y}^{(i)}\right)$ and $r_{X}^{(i)}=3 R_{X}^{(i+1)}$.

For every $i$ we express the set $f^{-1}\left(A_{i}\right)$ as the union of $k$ sets $\left\{B_{i}^{j}\right\}_{j=1}^{k}$ such that all $\left(r_{X}^{(i)}, r_{Y}^{(i)}\right)$-components of $B_{i}^{j}$ are $\left(R_{X}^{(i)}, R_{Y}^{(i)}\right)$-bounded for every $j$ and every point $x \in f^{-1}\left(A_{i}\right)$ belongs to at least $n$ sets.

Put $D_{i}^{j}=B_{i}^{j} \cap f^{-1}\left(U_{i}^{j}\right)$ and let $D^{j}$ be the union of all $D_{i}^{j}$. Notice $D^{j}$ 's cover $X$ by the use of Kolmogorov's argument: given $x \in X$ there is $i$ so that $f(x) \in A_{i}$. The set of $j$ 's such that $x \in B_{i}^{j}$ has at least $k-m$ elements, the set of $j$ 's such that $f(x) \in U_{i}^{j}$ has at least $k-n$ elements, so they cannot be disjoint.

Notice all $\left(r_{X}^{(i)}, r_{Y}^{(i)}\right)$-components of the set $D_{i}^{j}$ are $\left(R_{X}^{(i)}, R_{Y}^{(i)}\right)$-bounded. By 3.6 all $\left(r_{X}^{(n+1)}, r_{Y}^{(n+1)}\right)$-components of the set $D^{j}$ are $\left(3 R_{X}^{(1)}, 3 R_{Y}^{(1)}\right)$ bounded. Since $\left(r_{X}^{(n+1)}, r_{Y}^{(n+1)}\right)=\left(r, c_{f}(r)\right)$, by 3.3 all $r$-components of $D^{j}$ are $3 R_{X}^{(1)}$-bounded. Observe, $3 R_{X}^{(1)}$ is a linear function of $r$ (respectively, a dilation), if the coarseness control function $c_{f}$ of $f$, the $n$-dimensional control function of $Y$, and $D_{f}$ are linear (respectively, dilations).

Corollary 4.10. Suppose $f: X \rightarrow Y$ is a function of metric spaces and $m \geq 0$. asdim $(f) \leq m$ if and only if $f$ has an $m$-dimensional control function.

Proof. In one direction use 4.5. In the other direction apply 4.9 in the case of $n=0$.

Theorem 4.11.

$$
\operatorname{asdim}(X) \leq \operatorname{asdim}(f)+\operatorname{asdim}(Y)
$$


for any large scale uniform function $f: X \rightarrow Y$.

Proof. Follows from 4.9, 4.10, and 4.7.

In [3] there is a concept of a family $\left\{X_{\alpha}\right\}$ of subsets of $X$ satisfying $\operatorname{asdim}\left(X_{\alpha}\right) \leq n$ uniformly. Notice that in our language it means there is one function that serves as an $n$-dimensional control function for all $X_{\alpha}$.

Corollary 4.12 (Bell-Dranishnikov [3]). Let $f: X \rightarrow Y$ be a Lipschitz function of metric spaces. Suppose that, for every $R>0$,

$$
\operatorname{asdim}\left\{f^{-1}\left(B_{R}(y)\right)\right\} \leq n
$$

uniformly (in $y \in Y$ ). If $X$ is geodesic, then asdim $(X) \leq \operatorname{asdim}(Y)+n$.

Proof. $\operatorname{asdim}\left\{f^{-1}\left(B_{R}(y)\right)\right\} \leq n$ uniformly means $f$ has an $n$ dimensional control function, so apply 4.9 and 4.7 .

Remark 4.13. Notice Bell-Dranishnikov's version of Hurewicz type theorem 4.12 (see [3]) assumes that $X$ is geodesic. We do not use this assumption in 4.11 .

\section{Asymptotic Dimension of GROUPS}

J.Smith [27] showed that any two proper and left-invariant metrics on a given countable group $G$ are coarsely equivalent. We generalize that result as follows.

Proposition 5.1. Suppose $f: G \rightarrow H$ is a homomorphism of groups and $d_{G}, d_{H}$ are left-invariant metrics on $G$ and $H$, respectively. If $f:\left(G, d_{G}\right) \rightarrow\left(H, d_{H}\right)$ is coarsely proper (i.e., it sends bounded subsets of $G$ to bounded subsets of $H)$, then $f:\left(G, d_{G}\right) \rightarrow\left(H, d_{H}\right)$ is large scale uniform.

Proof. Suppose $r>0$. Since $f\left(B\left(1_{G}, r\right)\right)$ is bounded, there is $R>0$ such that $d_{H}\left(1_{H}, f(g)\right)<R$ for all $g \in B\left(1_{G}, r\right)$. If $x, y \in G$ satisfy $d_{G}(x, y)<r$, then $x^{-1} \cdot y \in B\left(1_{G}, r\right)$, so $d_{H}(f(x), f(y))=$ $d_{H}\left(1_{H}, f\left(x^{-1} y\right)\right)<R$.

Corollary 5.2. Suppose $f: G \rightarrow H$ is a homomorphism of groups and $d_{G}, d_{H}$ are left-invariant metrics on $G$ and $H$, respectively. If $d_{G}$ is proper, then $f:\left(G, d_{G}\right) \rightarrow\left(H, d_{H}\right)$ is large scale uniform.

Proof. Since bounded subsets of $G$ are finite, $f$ is coarsely proper.

Corollary 5.3 (J.Smith [27]). Any two proper left-invariant metrics on a group $G$ are coarsely equivalent. 
The subsequent result is derived in [16] from the corresponding theorem for finitely generated groups. We deduce it directly from 4.11.

Theorem 5.4 (Dranishnikov-Smith [16]). If $1 \rightarrow K \rightarrow G \rightarrow H \rightarrow 1$ is a short exact sequence of countable groups, then

$$
\operatorname{asdim}(G) \leq \operatorname{asdim}(K)+\operatorname{asdim}(H) .
$$

Proof. It suffices to show $\operatorname{asdim}(f)=k=\operatorname{asdim}(K)$, where $f: G \rightarrow$ $H$. Given $R_{H}>0$, the subspace $f^{-1}\left(B\left(1_{H}, R_{H}\right)\right)$ is in a bounded neighborhood of $K$ as $B\left(1_{H}, R_{H}\right)$ is finite, so it has the same asymptotic dimension as $K$. Fix the dimension control function $D\left(r_{G}, R_{H}\right)$ for that space. Notice that all spaces $f^{-1}\left(B\left(h, R_{H}\right)\right), h \in H$, are isometric to $f^{-1}\left(B\left(1_{H}, R_{H}\right)\right)$, so $D\left(r_{G}, R_{H}\right)$ is a dimension control function of all of them. By 4.10 , asdim $(f) \leq k$ and by applying 4.11 we are done.

In [16] the asymptotic dimension of a group $G$ is defined as the supremum of $\operatorname{asdim}(H), H$ ranging through all finitely generated subgroups of $G$. We provide an alternative definition which will be applied in the next section.

Definition 5.5. Given a finite subset $S$ of a group $G$, an $S$-component of $G$ is an equivalence class of $G$ of the relation $x \sim y$ iff $x$ can be connected to $y$ by a finite chain $x_{i}$ so that $x_{i}^{-1} \cdot x_{i+1} \in S$ for all $i$.

A family of subsets $\left\{A_{i}\right\}_{i \in J}$ is $S$-bounded if $x^{-1} \cdot y \in S$ for each $i \in J$ and all $x, y \in A_{i}$.

Proposition 5.6. Let $G$ be a group. $\operatorname{asdim}(G) \leq n$ if and only if for each finite subset $S$ of $G$ there is a finite subset $T$ of $G$ and a decomposition $G=A_{1} \cup \ldots \cup A_{n+1}$ such that $S$-components of $A_{i}$ are $T$-bounded for all $i \leq n+1$.

Proof. Suppose for each finite subset $S$ of $G$ there is a finite subset $T$ of $G$ and a decomposition $G=A_{1} \cup \ldots \cup A_{n+1}$ such that $S$-components of $A_{i}$ are $T$-bounded for all $i \leq n+1$. Given a countable subgroup $H$ of $G$ and given a proper left-invariant metric $d_{H}$ on $H$, we put $S=B\left(1_{H}, r\right)$ and $R=\max \left\{d_{H}\left(1_{H}, t\right) \mid t \in T \cdot T \cap H\right\}$ (by $T \cdot T$ we mean all products $t \cdot s$, where $t, s \in T)$. Notice that $r$-components of $A_{i} \cap H$ are $R$-bounded.

Conversely, suppose $\operatorname{asdim}(H) \leq n$ for all finitely generated subgroups $H$ of $G$. Given a finite subset $S$ of $G$ let $H$ be the subgroup of $G$ generated by $S$ and let $d$ be the word metric on $H$ induced by $S$. Since $\operatorname{asdim}(H) \leq n$, there is a decomposition of $H$ into $A_{1} \cup \ldots \cup A_{n+1}$ such that 1-components of $A_{i}$ are $m$-bounded for all $i \leq n+1$ and some $m>0$. Let $T=B\left(1_{H}, m+1\right)$. Pick a representative $g_{j} \in G, j \in J$, of 
each left coset of $H$ in $G$. Put $B_{i}=\bigcup_{j \in J} g_{j} \cdot A_{i}$ and notice $S$ components of $B_{i}$ are $T$-bounded.

\section{LinEARLY CONTROLLED ASYMPTOTIC DIMENSION OF GROUPS}

Unlike the asymptotic dimension of countable groups, the asymptotic Assouad-Nagata dimension may depend on the left-invariant metric. Piotr Nowak [23] constructed finitely generated groups $G_{n}$ of asymptotic dimension $n \geq 2$ and $\operatorname{asdim}_{A N}\left(G_{n}\right)=\infty$. Obviously, there is no such example for $n=0$ as finitely generated groups of asymptotic dimension 0 are finite. However, 6.4 does provide a countable group $G$ with a proper, left-invariant metric $d$ such that $\operatorname{asdim}(G, d)=0$ and $\operatorname{asdim}_{A N}(G, d)=\infty$.

The purpose of this section is to solve in negative the following two problems of Dranishnikov [14].

Problem 6.1. Does l-asdim $(X)=\operatorname{asdim}_{A N}(X)$ hold for metric spaces?

Problem 6.2. Find a metric space $X$ of minimal $\operatorname{asdim}(X)$ such that $\operatorname{asdim}(X)<1-\operatorname{asdim}(X)$.

Here is an answer to 6.2.

Proposition 6.3. There is a proper, left-invariant metric $d_{G}$ on $G=$ $\bigoplus_{n=2}^{\infty} \mathbb{Z} / n$ such that l-asdim $\left(G, d_{G}\right)>0=\operatorname{asdim}\left(G, d_{G}\right)$.

Proof. Pick a generator $g_{n}$ of $\mathbb{Z} / n$ and assign it the norm of $n$ for $n \geq 2$. Extend the norm over all elements $g$ of $G$ as the minimum of $\sum_{n=1}^{\infty}\left|k_{n}\right| \cdot n$, where $g=\sum_{n=1}^{\infty} k_{n} \cdot g_{n}$ and $k_{n}$ are integers. Notice that $d_{G}(g, h):=|g-h|$ is a proper and invariant metric on $G$. Suppose there is an unbounded subset $U$ of $\mathbb{R}_{+}$such that for some $C>0$ all $r$-components of $G$ are $(C \cdot r)$-bounded for $r \in U$. Pick $r \in U$ such that $4 C+4<r$ and let $n$ be an integer such that $r-2<2 n \leq r$. Notice all $2 n$-components of $G$ are contained in some $r$-components of $G$, so they are $(n-1) \cdot(n+1)$-bounded as $C<\frac{r-4}{4} \leq \frac{n-2}{2}$ and $r<2 n+2$. However, the $2 n$-component of 0 contains $\mathbb{Z} / 2 n$ and $\left|n \cdot g_{2 n}\right|=2 n^{2}>$ $(n-1) \cdot(n+1)$, a contradiction.

Proposition 6.4. There is an Abelian torsion group $G$ with a proper invariant metric $d_{G}$ such that $l$-asdim $\left(G, d_{G}\right)=0$ and $\operatorname{asdim}_{A N}\left(G, d_{G}\right)=$ $\infty$.

Proof. Consider $\mathbb{Z}^{n+1}$ with the standard word metric $\rho_{n}$. Since $\operatorname{asdim}\left(\mathbb{Z}^{n+1}\right)>n$, there is $r(n)>0$ such that any decomposition of 
$\mathbb{Z}^{n+1}$ as the union $X_{0} \cup \ldots \cup X_{n}$ forces one $X_{i}$ to have $r(n)$-components without a common upper bound. Without loss of generality we may assume $r(n)$ is an integer greater than $n$. Put $s(n)=8 \cdot n \cdot r(n)$ and consider $G_{n}=\left(\mathbb{Z}_{s(n)}\right)^{n+1}$ with the metric $d_{n}$ equal to $t(n)$ times the standard word metric. Numbers $t(n)$ are chosen so that $t(n+1)>$ $\operatorname{diam}\left(G_{1}\right)+\ldots+\operatorname{diam}\left(G_{n}\right)$ for all $n \geq 1$. Since $\operatorname{diam}\left(G_{n}\right)=4 n t(n) r(n)$, we want $t(n+1)=1+4 \cdot t(1) r(1)+\ldots+4 n \cdot t(n) r(n)$ for all $n \geq 1$.

The group $G$ is the direct sum of all $G_{n}$ with the obvious metric $d$ : $d\left(\left\{x_{n}\right\},\left\{y_{n}\right\}\right)=\sum d_{n}\left(x_{n}, y_{n}\right)$. Notice that $\frac{t(n)}{2}$-components of $G$ are of size at most $t(n)$. Indeed, any $\frac{t(n)}{2}$-component of $G$ is contained in $x+G_{1} \oplus \ldots \oplus G_{n-1}$ for some $x \in G$. $\operatorname{Thus} \operatorname{l-asdim}(G, d)=0$.

Consider the projection $\pi_{n}: \mathbb{Z}^{n+1} \rightarrow G_{n}$ and notice it is $t(n)$-Lipschitz. Moreover, if two points $x$ and $y$ in $\mathbb{Z}^{n+1}$ satisfy $\rho_{n}(x, y) \leq 4 n r(n)$, then $d_{n}(x, y)=t(n) \cdot \rho_{n}(x, y)$.

Suppose $G_{n}$ equals $Y_{0} \cup \ldots \cup Y_{n}$. Assume $r(n)$-components of some $X_{j}=\pi_{n}^{-1}\left(Y_{j}\right)$ are not uniformly bounded. Therefore there is a sequence $x_{0}, \ldots, x_{k}$ in $X_{j}$ such that $\rho_{n}\left(x_{i}, x_{i+1}\right)<r(n)$ and $(n+2) \cdot r(n)>$ $\rho_{n}\left(x_{0}, x_{k}\right)>n \cdot r(n)$. Assume such a sequence does not exist with number of elements smaller than $k+1$. Therefore $d_{n}\left(\pi_{n}\left(x_{i}\right), \pi_{n}\left(x_{i+1}\right)\right)=$ $t(n) \cdot \rho_{n}\left(x_{i}, x_{i+1}\right)<t(n) \cdot r(n)$ and $(n+2) \cdot t(n) r(n)>\rho_{n}\left(\pi_{n}\left(x_{0}\right), \pi_{n}\left(x_{k}\right)\right)>$ $n \cdot t(n) r(n)$. Thus, one $(t(n) \cdot r(n))$-component of $Y_{i}$ is of diameter bigger than $n \cdot t(n) r(n)$.

Suppose $\operatorname{asdim}_{A N}(G, d)=k<\infty$ and $D_{G}(r)=c \cdot r+b$ is a $k$ dimension control function of $(G, d)$. Pick $n>\max (k,|c|,|b|)+2$ and choose a decomposition $Y_{0} \cup \ldots \cup Y_{k}$ of $G$ so that $(t(n) \cdot r(n))$-components of each $Y_{i}$ are bounded by $c \cdot t(n) \cdot r(n)+b<(c \cdot t(n)+1) \cdot r(n)$. Notice there is a 1-Lipschitz projection $p_{n}: G \rightarrow G_{n}$. Therefore each set $p_{n}\left(Y_{i}\right)$ has $(t(n) \cdot r(n))$-components bounded by $(c \cdot t(n)+1) \cdot r(n)$. On the other hand, for some $j$, there is an $(t(n) \cdot r(n))$-component of $p_{n}\left(Y_{j}\right)$ of diameter bigger than $n t(n) \cdot r(n)$. Thus, $c \cdot t(n)+1>n t(n)$ and $c>n-1 / t(n)$, a contradiction.

Remark 6.5. Proposition 6.4 solves Problem 6.1 in negative.

Proposition 6.6. For any countable group $G$ there is a proper leftinvariant metric $d_{G}$ such that $\operatorname{asdim}_{A N}\left(G, d_{G}\right)=\operatorname{asdim}(G)$.

Proof. Let $\operatorname{asdim}(G)=n-1$. Express $G$ as the union of an increasing sequence $S_{0} \subset S_{1} \ldots$ of its finite subsets so that $\left\{1_{G}\right\}=S_{0}$, each $S_{i}$ is symmetric, and for each $i$ there is a decomposition of $G$ as $A_{1}^{i} \cup \ldots \cup A_{n}^{i}$ so that $S_{i}$-components of $A_{k}^{i}$ are $S_{i+1}$-bounded. Define $d_{G}(x, y)$ as the smallest $i$ so that $x^{-1} \cdot y \in S_{i}$. Notice that in the metric $d_{G}$ all $i$-components of $A_{k}^{i}$ are $(i+1)$-bounded. 


\section{Assouad-Nagata dimension}

Definition 7.1. Given a function $f: X \rightarrow Y$ of metric spaces we define the Assouad-Nagata dimension $\operatorname{dim}_{A N}(f)$ of $f$ as the minimum of $m$ for which there is an $m$-dimensional control function $D_{f}\left(r_{X}, R_{Y}\right)$ of the form $a \cdot r_{X}+b \cdot R_{Y}$.

Theorem 7.2. If $f: X \rightarrow Y$ is a Lipschitz function of metric spaces, then $\operatorname{dim}_{A N}(X) \leq \operatorname{dim}_{A N}(f)+\operatorname{dim}_{A N}(Y)$.

Proof. Notice in the proof of 4.9 the resulting dimensional control function of $X$ is a dilation if $c_{f}, D_{X}$, and $D_{f}$ are dilations.

Remark 7.3. Notice the proof of of 4.9 can be analyzed to give a version of 7.2 for microscopic Assouad-Nagata dimension.

\section{Asymptotic Assouad-Nagata dimension}

Definition 8.1. Given a function $f: X \rightarrow Y$ of metric spaces we define the asymptotic Assouad-Nagata dimension $\operatorname{asdim}_{A N}(f)$ of $f$ as the minimum of $m$ for which there is an $m$-dimensional control function $D_{f}\left(r_{X}, R_{Y}\right)$ of the form $a \cdot r_{X}+b \cdot R_{Y}+c$.

Theorem 8.2. If $f: X \rightarrow Y$ is an asymptotically Lipschitz function of metric spaces, then $\operatorname{asdim}_{A N}(X) \leq \operatorname{asdim}_{A N}(f)+\operatorname{asdim}_{A N}(Y)$.

Proof. Notice in the proof of 4.9 the resulting dimensional control function of $X$ is linear if $c_{f}, D_{X}$, and $D_{f}$ are linear.

Proposition 8.3. Let $n \geq 0$. If $\left(G, d_{G}\right)$ is a group equipped with a proper, left-invariant metric $d_{G}$, then the following conditions are equivalent:

a. $\operatorname{asdim}_{A N}\left(G, d_{G}\right) \leq n$.

b. There are constants $C, M>0$ such that the function $r \rightarrow M \cdot r+$ $C$ is an $n$-dimensional control function for all finitely generated subgroups of $G$.

Proof. a) $\Longrightarrow$ b) follows from the proof of 3.10 . b) $\Longrightarrow$ a) is obvious.

Proposition 8.4. If $1 \rightarrow K \rightarrow G \rightarrow H \rightarrow 1$ is an exact sequence and $G$ is a finitely generated group, then there are word metrics $d_{G}$ on $G$ and $D_{H}$ on $H$ such that $f:\left(G, d_{G}\right) \rightarrow\left(H, d_{H}\right)$ is 1-Lipschitz and for any $m$-dimensional control function $D_{K}$ on $K$ the function

$$
D_{f}\left(r_{G}, R_{H}\right):=D_{K}\left(r_{G}+2 R_{H}\right)+2 R_{H}
$$

is an $m$-dimensional control function of $f$. 
Proof. Let $S$ be a symmetric finite set of generators for $G$. Let $d_{G}$ be the word metric on $G$ induced by $S$ and let $d_{H}$ be the word metric on $H$ induced by $f(S)$.

Let $B=B\left(1_{H}, R_{H}\right)$ and $A=f^{-1}(B)$. Notice $A \subset B\left(K, R_{H}\right)$. Indeed, if $d_{H}\left(1_{H}, f(a)\right)<i=R_{H}$, then $f(a)=\prod_{i=1}^{m} f\left(s_{i}\right)$ such that $k<R_{H}$ and $s_{i} \in S$ for $i \leq m$. Consequently, $a=k \cdot x$ so that $k \in K$ and $x=\prod_{i=1}^{m} s_{i}$ has the property $d_{G}\left(1_{G}, x\right)<R_{H}$.

Now $d_{G}(k \cdot s, k)=d_{G}\left(s, 1_{G}\right) \leq i-1<i$ and $a \in B\left(K, R_{H}\right)$. By 3.7, the function $D_{f}\left(r_{G}, R_{H}\right):=D_{K}\left(r_{G}+2 R_{H}\right)+2 R_{H}$ is an $m$-dimensional control function of $B\left(K, R_{H}\right)$. Since all $f^{-1}\left(B\left(y, R_{H}\right)\right)$ are isometric to $A$, we are done.

Corollary 8.5. If $1 \rightarrow K \rightarrow G \rightarrow H \rightarrow 1$ is an exact sequence of groups so that $G$ is finitely generated, then

$$
\operatorname{asdim}_{A N}\left(G, d_{G}\right) \leq \operatorname{asdim}_{A N}\left(K, d_{G} \mid K\right)+\operatorname{asdim}_{A N}\left(H, d_{H}\right)
$$

for any word metrics metrics $d_{G}$ on $G$ and $d_{H}$ on $H$.

Proof. Use the metrics as in 8.4. That way

$$
\operatorname{asdim}_{A N}(f) \leq \operatorname{asdim}_{A N}\left(K, d_{G} \mid K\right),
$$

so applying 8.2 one gets the desired inequality.

One is tempted to define the linear asymptotic dimension of arbitrary groups as the supremum of $\operatorname{asdim}_{A N}(H)$ for all finitely generated subgroups $H$ of $G$, However, one runs into problems with that definition.

Question 8.6. Suppose $G$ is a finitely generated group and $H$ is its finitely generated subgroup. Does $\operatorname{asdim}_{A N}(H) \leq \operatorname{asdim}_{A N}(G)$ hold? Is there a case of $\operatorname{asdim}_{A N}(H)=\infty$ and $\operatorname{asdim}_{A N}(G)<\infty$ ?

Question 8.7. Suppose $G$ is a finitely generated group and $H$ is its finitely generated subgroup. Does $\operatorname{asdim}_{A N}(H)=\operatorname{asdim}_{A N}\left(H, d_{G} \mid H\right)$ hold for any word metric $d_{G}$ on $G$ ?

Question 8.8. Suppose $G$ and $H$ are two finitely generated groups of finite asymptotic Assouad-Nagata dimension. Is $\operatorname{asdim}_{A N}(G * H)$ finite?

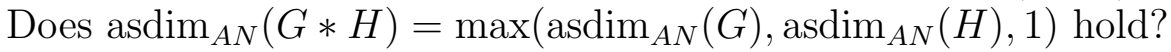

To complete this section we state and prove a version of the the Hurewicz theorem for groups acting on spaces of finite asymptotic Assouad-Nagata dimension. For this we need the concept of $R$-stabilizers. Let $G$ act on the metric space $X$ by isometries and let $R>0$. Given 
$x_{0} \in X$ the $R$-stabilizer of $x_{0}$ is defined by $W_{R}\left(x_{0}\right)=\{\gamma \in G$ : $\left.d\left(\gamma \cdot x_{0}, x_{0}\right) \leq R\right\}$.

Theorem 8.9. Let $G$ be a finitely generated group acting by isometries on a metric space $X$ of finite asymptotic Assouad-Nagata dimension. Fix a point $x_{0} \in X$. If there are constants $m, b, c>0$ such that $r \rightarrow$ $m \cdot r+b \cdot R+c$ is a $k$-dimensional control function of $W_{R}\left(x_{0}\right)$ for all $R$, then

$$
\operatorname{asdim}_{A N}(G) \leq k+\operatorname{asdim}_{A N}(X) .
$$

Proof. Let $S$ be a symmetric generating set for $G$ and let $\lambda=$ $\max \left\{d_{X}\left(s \cdot x_{0}, x_{0}\right) \mid s \in S\right\}$. Define $\pi: G \rightarrow X$ by $\pi(\gamma)=\gamma \cdot x_{0}$ and notice that $\pi$ is $\lambda$-Lipschitz. Also, $\pi^{-1}\left(B_{R}\left(g \cdot x_{0}\right)\right)=g W_{R}\left(x_{0}\right)$ shows that the sets $\left(\pi^{-1}\left(B_{R}\left(g \cdot x_{0}\right)\right)\right)$ are all isometric to $W_{R}\left(x_{0}\right)$ and we can use 8.2 to get our result.

\section{REFERENCES}

[1] P. Assouad, Sur la distance de Nagata, C. R. Acad. Sci. Paris Ser. I Math. 294 (1982), no. 1, 31-34.

[2] P. Assouad, Plongements lipschitziens dans $R^{n}$, Bull. Soc. Math. France 111 (1983), 429-448.

[3] G. Bell and A. Dranishnikov, A Hurewicz-type theorem for asymptotic dimension and applications to geometric group theory, Preprint, math.GR/0407431 (2004).

[4] G. Bell and A. Dranishnikov, Asymptotic dimension in Bedlewo, preprint

[5] G.Bell and A.Dranishnikov, On asymptotic dimension of groups acting on trees, Geom. Dedicata 103 (2004), 89-101.

[6] N. Brodskiy, J. Dydak, Coarse dimensions and partitions of unity, preprint math.GT/0506547.

[7] N. Brodskiy, J. Dydak, J. Higes, A. Mitra, Assouad-Nagata dimension via Lipschitz extensions, preprint math.MG/0601226.

[8] N.Brodskiy, J.Dydak, J.Higes, A.Mitra, Dimension zero at all scales, in preparation.

[9] S. Buyalo, Asymptotic dimension of a hyperbolic space and capacity dimension of its boundary at infinity, Algebra i analis (St. Petersburg Math. J.), 17 (2005), 70-95 (in Russian).

[10] S. Buyalo, A. Dranishnikov and V. Schroeder, Embedding of hyperbolic groups into products of binary trees, preprint.

[11] S. Buyalo, N. Lebedeva, Dimension of locally and asymptotically self-similar spaces, preprint math.GT/0509433.

[12] G. Carlsson and B. Goldfarb, On homological coherence of discrete groups.

[13] A.Dranishnikov, Asymptotic topology, Russian Math. Surveys 55 (2000), no.6, $1085-1129$.

[14] A.Dranishnikov, private comminication.

[15] A.Dranishnikov and T.Januszkiewicz, Every Coxeter group acts amenably on a compact space, Proceedings of the 1999 Topology and Dynamics Conference (Salt Lake City, UT). Topology Proceedings 24 (1999), 135-141. 
[16] A.Dranishnikov and J.Smith, Asymptotic dimension of discrete groups, Fund. Math. 189 (2006), 27-34.

[17] A. Dranishnikov and M.Zarichnyi, Universal spaces for asymptotic dimension, Topology and its Appl. 140 (2004), no.2-3, 203-225.

[18] R. Engelking, Theory of dimensions finite and infinite, Sigma Series in Pure Mathematics, vol. 10, Heldermann Verlag, 1995.

[19] M. Gromov, Asymptotic invariants for infinite groups, in Geometric Group Theory, vol. 2, 1-295, G.Niblo and M.Roller, eds., Cambridge University Press, 1993.

[20] U. Lang, T. Schlichenmaier, Nagata dimension, quasisymmetric embeddings, and Lipschitz extensions, arXive:math. MG/0410048 (2004).

[21] J. Nagata, Note on dimension theory for metric spaces, Fund. Math. 45 (1958) $143-181$.

[22] J. Nagata, Modern Dimension Theory, North-Holland 1965.

[23] P.W.Nowak, On exactness and isoperimetric profiles of discrete groups, preprint.

[24] P. Ostrand, A conjecture of J. Nagata on dimension and metrization, Bull. Amer. Math. Soc. 71 (1965), 623-625.

[25] P.A. Ostrand, Dimension of Metric Spaces and Hilbert's problem 13, Bull. Amer. Math. Soc. 71 (1965), 619622.

[26] J. Roe, Lectures on coarse geometry, University Lecture Series 31, American Mathematical Society, Providence, RI, 2003.

[27] J. Smith, On Asymptotic Dimension of Countable Abelian Groups, preprint math.GR/0504447.

[28] Yaki Sternfeld, Hilbert's 13th Problem and Dimension, Geometrical aspects of functional analysis, (J. Lindenstrauss and V. Milman, editors) Notes in Math. 1376, Springer-Verlag, Berlin, 1987-8, 1-49.

University of Tennessee, Knoxville, TN 37996, USA

E-mail address: brodskiy@math.utk.edu

University of Tennessee, Knoxville, TN 37996, USA

E-mail address: dydak@math.utk.edu

Departament of Mathematics, Ben-Gurion University of the Nagev, P.O.B. 653, BeER-Sheba 84105, Israel.

E-mail address: mlevine@math.bgu.ac.il

University of Tennessee, Knoxville, TN 37996, USA

E-mail address: ajmitra@math.utk.edu 